\title{
Helicobacter cinaedi Hepatic Cyst Infection with Bacteremia
}

\section{Tetsuya Suzuki, Satoshi Kutsuna, Motoyuki Tsuboi, Masayuki Ota, Kayoko Hayakawa, Norio Ohmagari}

Author affiliation: National Center for Global Health and Medicine, Tokyo, Japan

DOI: https://doi.org/10.3201/eid2503.180936

Helicobacter cinaedi is an enterohepatic bacillus that causes infections of various manifestations. We report a novel case of hepatic cyst infection with bacteremia caused by $\mathrm{H}$. cinaedi in an immunocompetent woman in Japan. Further research is warranted to identify the epidemiologic and clinical features of $H$. cinaedi infection.

$H$ elicobacter cinaedi is a gram-negative, spiral-shaped enterohepatic bacillus found in the digestive tracts of humans and other animals (1). Many reports have described that $H$. cinaedi can cause infections in immunocompromised patients (2). In recent decades, however, several cases of immunocompetent patients with $H$. cinaedi infection have been reported (3). Although various manifestations of $H$. cinaedi infection have been described, to our knowledge, no cases of hepatic cyst infection have been reported. We report a case illustrating an $H$. cinaedi hepatic cyst infection with bacteremia in an immunocompetent patient in Japan.

In July 2017, a 73-year-old woman was referred to the National Center for Global Health and Medicine (Shinju$\mathrm{ku}$, Japan) because of a 2-day history of abdominal pain, vomiting, and fever. She had schizophrenia and had been hospitalized in a psychiatric hospital for $>20$ years. At admission, her body temperature was $37.5^{\circ} \mathrm{C}$, and other vital signs were stable. Physical examination revealed a slight tenderness on her abdomen with mild rebound tenderness. Her laboratory findings showed elevated leukocytes $(10,170$ cells $/ \mathrm{mL}$, reference range 3,300-8,600 cells $/ \mu \mathrm{L})$, neutrophils $(87 \%$, reference $40 \%-71 \%)$, and C-reactive protein $(11.7 \mathrm{mg} / \mathrm{dL}$, reference $0-0.14 \mathrm{mg} /$ $\mathrm{dL})$. Total bilirubin $(0.8 \mathrm{mg} / \mathrm{dL})$, aspartate aminotransferase $(13 \mathrm{U} / \mathrm{L})$, alanine aminotransferase $(10 \mathrm{U} / \mathrm{L})$, alkaline phosphatase (264 U/L), and gamma-glutamyl transferase (21 U/L) levels were within reference ranges. Enhanced computed tomography (CT) showed slight ascites, but no apparent signs of bowel obstruction. We found a large hepatic cyst ( $63 \mathrm{~mm}$ in diameter) on the left liver lobe (Figure); the cyst had been $44 \mathrm{~mm}$ in diameter a month earlier, when she was hospitalized because of a small intestinal obstruction.
On the day of hospital admission, we obtained 2 blood culture samples and started the patient on a regimen of piperacillin/tazobactam. Her fever persisted. On day 5, blood culture results were positive for spiral-shaped gramnegative rod bacteria in both sets of aerobic bottles (after $80.6 \mathrm{~h}$ and $104.5 \mathrm{~h}$ ) (BACTEC, Becton, Dickinson and Company, https://www.bd.com). Enhanced CT on day 5 revealed enlargement of the hepatic cyst from $63 \mathrm{~mm}$ to $69 \mathrm{~mm}$ in diameter. The patient's tenderness was most pronounced in the upper abdomen. We suspected hepatic cyst infection and placed an ultrasound-guided drainage tube, by which we obtained $\approx 100 \mathrm{~mL}$ of purulent liquid. The patient's fever subsided rapidly, within 1 day after drainage.

We identified the isolates from the blood cultures as $H$. cinaedi by using matrix-assisted laser desorption/ionization-time of flight mass spectrometry (score 2.07). Subsequent PCR testing of pus obtained by drainage also gave a positive result for $H$. cinaedi.

We collected $>100 \mathrm{~mL}$ of pus in the first 2 drainage days (hospital days 5-6). The patient showed defervescence on day 6 . We changed her antibiotic to ampicillin/ sulbactam on day 10. A nonenhanced CT scan indicated that the hepatic cyst had collapsed from drainage on day 17. We removed the drainage tube on day 18 and stopped the antibiotic regimen on day 22. The patient's condition was stable after drainage, and she was discharged to the previous psychiatric hospital on day 24.

We report a novel case of hepatic cyst infection caused by $H$. cinaedi. Uwamino et al. reported a retrospective observational study of community-acquired $H$. cinaedi bacteremia (3), showing that cellulitis was the most common manifestation in community-acquired cases, whereas bacteremia without any specific focus was the leading type of infection in healthcare-associated and nosocomial settings. In the case we describe, the patient was in a nosocomial setting at the onset. Thus, primary occult bacteremia is the

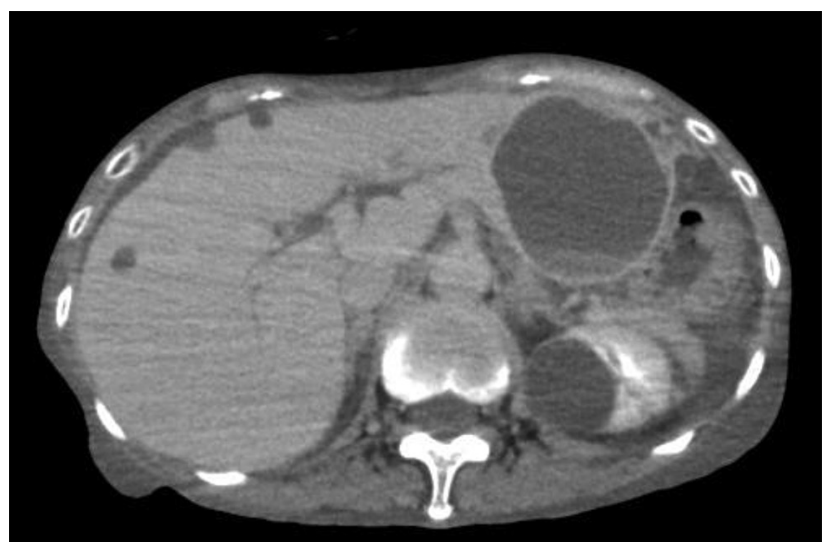

Figure. Enhanced computed tomography image showing a large hepatic cyst ( $63 \mathrm{~mm}$ in diameter) on the left liver lobe in a patient with Helicobacter cinaedi infection on the day of hospital admission, Tokyo, Japan, July 2017. 
leading hypothesis. However, H. cinaedi is an enterohepatic bacterium, and the patient had undergone surgery for an adhesive small intestinal obstruction 1 month before. Her medical and surgical history might have increased the intraintestinal pressure and induced the hepatic cyst infection through biliary reflux.

The patient had schizophrenia but was not immunocompromised beyond her surgical history. Many cases of $H$. cinaedi infection have been reported in immunocompromised hosts (2), but reports of $H$. cinaedi infections in immunocompetent patients have been increasing (3). Matsumoto et al. showed that $H$. cinaedi bacteremia was found in only $0.06 \%$ of total blood samples (4); none of the patients in their study were HIV-positive, but many were immunocompromised by other conditions. Kiehlbauch et al. also conducted a retrospective study of $H$. cinaedi bacteremia and found that $45 \%$ of patients were HIV-positive (5). H. cinaedi infection can occur regardless of a patient's immunologic or environmental status.

$H$. cinaedi infections are often reported in Japan. Miyake et al. reported that the $H$. cinaedi detection rate has increased after introduction of the BACTEC system (6). We also used BACTEC bottles. The widespread use of this type of blood culture bottle throughout Japan might contribute to the positivity rate of $H$. cinaedi.

We report a case of $H$. cinaedi hepatic cyst infection with bacteremia. $H$. cinaedi infection can occur in both nosocomial and community-acquired situations and in both immunocompromised and immunocompetent patients; its manifestations vary quite widely. Although the positivity rate of $H$. cinaedi is very low, it might still be overlooked. Further research is warranted to identify the epidemiologic and clinical features of $H$. cinaedi infection.

\section{About the Author}

Dr. Suzuki is a medical doctor at the National Center for Global Health and Medicine in Shinjuku-ku, Tokyo, Japan. His main research interest is nosocomial infectious diseases.

\section{References}

1. Kawamura Y, Tomida J, Morita Y, Fujii S, Okamoto T, Akaike T. Clinical and bacteriological characteristics of Helicobacter cinaedi infection. J Infect Chemother. 2014;20:517-26. http://dx.doi.org/ 10.1016/j.jiac.2014.06.007

2. Kamimura K, Kumaki D, Arita M, Kobayashi Y, Mizuno K, Kusama F, et al. First case of bacteremia caused by Helicobacter cinaedi in a patient with liver cirrhosis: a case report and literature review. Clin J Gastroenterol. 2015;8:306-17. http://dx.doi.org/10.1007/s12328-015-0600-0

3. Uwamino Y, Muranaka K, Hase R, Otsuka Y, Hosokawa N. Clinical features of community-acquired Helicobacter cinaedi bacteremia. Helicobacter. 2016;21:24-8. http://dx.doi.org/10.1111/ hel.12236

4. Matsumoto T, Goto M, Murakami H, Tanaka T, Nishiyama H, Ono E, et al. Multicenter study to evaluate bloodstream infection by Helicobacter cinaedi in Japan. J Clin Microbiol. 2007;45:28537. http://dx.doi.org/10.1128/JCM.00465-07

5. Kiehlbauch JA, Tauxe RV, Baker CN, Wachsmuth IK. Helicobacter cinaedi-associated bacteremia and cellulitis in immunocompromised patients. Ann Intern Med. 1994;121:90-3. http://dx.doi.org/1 0.7326/0003-4819-121-2-199407150-00002

6. Miyake N, Chong Y, Nishida R, Nagasaki Y, Kibe Y, Kiyosuke M, et al. A dramatic increase in the positive blood culture rates of Helicobacter cinaedi: the evidence of differential detection abilities between the Bactec and BacT/Alert systems. Diagn Microbiol Infect Dis. 2015;83:232-3. http://dx.doi.org/ 10.1016/j.diagmicrobio.2015.07.017

Address for correspondence: Satoshi Kutsuna, National Center for Global Health and Medicine, Disease Control and Prevention Center, 1-21-1, Toyama, Shinjuku-ku, Tokyo, 162-8655, Japan; email: sonare.since1192@gmail.com

\section{Mycobacterium tuberculosis RD-Rio Strain in Kazakhstan}

\author{
Yuriy Skiba, Igor Mokrousov, Dilyara Nabirova, \\ Anna Vyazovaya, Elina Maltseva, \\ Natalya Malakhova, Gulnara Ismagulova, \\ Ilva Pole, Renate Ranka, Zhanar Sapiyeva, \\ Shakhimurat Ismailov, Daphne Moffett
}

Author affiliations: Almaty Branch of National Center for

Biotechnology at Central Reference Laboratory, Almaty, Kazakhstan (Y. Skiba); Aitkhozhin Institute of Molecular Biology and Biochemistry, Almaty (Y. Skiba, E. Maltseva, N. Malakhova, G. Ismagulova); St. Petersburg Pasteur Institute, St. Petersburg, Russia (I. Mokrousov, A. Vyazovaya); Centers for Disease Control and Prevention, Central Asia Regional Office, Almaty (D. Nabirova, D. Moffett); Latvian Biomedical Research and Study Centre, Riga, Latvia (I. Pole, R. Ranka); Ministry of Health, Almaty (Z. Sapiyeva, S. Ismailov); The Global Fund, Geneva, Switzerland (S. Ismailov)

DOI: https://doi.org/10.3201/eid2503.181179

Mycobacterium tuberculosis RD-Rio strains are still rare in the former Soviet Union countries and Asia. We describe a strain in Kazakhstan that belongs to the RD-Rio secondary branch, which is endemic to northwest Russia and eastern Europe. Although RD-Rio strains are frequently multidrug resistant, this heterogeneous branch included only drugsusceptible isolates. 